THE HIGH CONTACT PRINCIPLE IN OPTIMAL STOPPING

\title{
AND STOCHASTIC WAVES
}

\section{Bernt $\emptyset \mathrm{ksendal}{ }^{*}$}

\section{SUMMARY}

The high contact principle in optimal stopping states that at the boundary $\partial D$ of the continuation region $D$ the reward function $g$ has a smooth fit with the optimal expected reward function $g^{*}$, in the sense that

$$
\begin{gathered}
g=g^{*} \text { on } \partial D \\
\nabla g=\nabla g^{*} \text { on } \partial D
\end{gathered}
$$

Thus this principle gives the crucial link between optimal stopping and free boundary problems.

If the system is described by Brownian - or geometric Brownian - motion then it is easy to prove the high contact principle. However, in the general case when the system is described by a diffusion in $\mathbb{R}^{n}$ all the proofs in the literature known to the author are long and complicated.

The purpose of this work is to show how a result by Dynkin and Vanderbei about stochastic waves can be used to give a short proof of the high contact principle. Moreover, this proof works under weaker conditions than known before. 
THE HIGH CONTACT PRINCIPLE IN OPTIMAL STOPPING

\section{AND STOCHASTIC WAVES}

Bernt $\emptyset \mathrm{ksendal}$

\section{§1. Introduction}

The (diffusion) optimal stopping problem can be formulated as follows:

Let $\left(X_{t}, \mathcal{M}_{t}, Q^{x}, \theta_{t}\right)$ be an Ito diffusion, i.e. the (strong) solution of an Ito stochastic differential equation in $\mathbb{R}^{n}$ :

$$
d X_{t}=b\left(X_{t}\right) d t+\sigma\left(X_{t}\right) d B_{t}
$$

where $b: \mathbb{R}^{n} \rightarrow \mathbb{R}^{n}$ and $\sigma: \mathbb{R}^{n} \rightarrow \mathbb{R}^{n \times m}$ (where $\mathbb{R}^{n \times m}$ denotes the $n \times m$ matrices with real entries) are given Lipschitz functions with at most linear growth and $\left(B_{t}, \mathcal{F}_{t}, P^{x}, \theta_{t}\right)$ denotes $m$-dimensional Brownian motion. Let $g$ be a given non-negative (or lower bounded) continuous function on $\mathbb{R}^{n}$. The optimal stopping problem is to find $g^{*}$ and an $\mathcal{F}_{t}$-stopping time $\tau^{*}$ such that

$$
g^{*}(x):=\sup _{\tau} E^{x}\left[g\left(X_{t}\right)\right]=E^{x}\left[g\left(X_{\tau *}\right)\right]
$$

where $E^{x}$ denotes expectation w.r.t. the law $Q^{x}$ of $X_{t}$ starting at $x$, the sup being taken over all $\mathcal{F}_{t}$-stopping times $\tau$. (If $\tau(w)=\infty$ then $g\left(X_{\tau}(w)\right.$ ) is interpreted as 0 ). (The similar problem involving "inf" instead of "sup" in (1.2) can be transformed to (1.2) by changing sign on $g$, at least if $g$ is bounded.)

The function $g$ is often called the reward function. Thus we interpret $g\left(X_{\tau}\right)$ as the reward obtained by stopping $X_{t}$ at time $t=\tau$. The problem is therefore to find a stopping time $\tau^{*}$ which maximizes the expected reward and to find this maximal reward $g^{*}$.

A fundamental result in optimal stopping is that $\tau^{*}$ can be realized as the first exit time $\tau_{D}$ for $X_{t}$ from a certain region $D$ (see for example [10, Theorem 10.9] for details):

THEOREM A (Optimal stopping theorem)

Let $\hat{g}$ denote the least superharmonic majorant of $g$. Then

$$
g^{*}=\hat{g}
$$


Define

$$
D=\{x ; g(x)<\hat{g}(x)\} \quad \text { (the continuation region) }
$$

Assume that $\tau_{D}:=\inf \left\{t>0 ; X_{t} \notin D\right\}$ is finite a.s. $Q^{x}$ and that the family

$$
\left\{g\left(X_{\tau}\right)\right\}_{\tau \leq \tau_{D}} \text { is uniformly } Q^{x} \text { - integrable. }
$$

(For example, it suffices that $g$ is bounded). Then

$$
g^{*}(x)=E^{x}\left[g\left(X_{\tau_{D}}\right)\right]
$$

so $\tau^{*}=\tau_{D}$ is optimal.

Thus $g^{*}=\hat{g}$ coincides with $g$ outside $D$. The high contact principle states that - under certain conditions - the contact between $g$ and $g^{*}$ on $\partial D$ is smooth, in the sense that

$$
\lim _{\substack{x \rightarrow y \\ x \in D}} \nabla g^{*}(x)=\nabla g(y) \text { for } y \in \partial D
$$

This result is crucial for the connection between optimal stopping problems and free boundary problems.

The first time this principle was formulated seems to be in a paper by Samuelson [11], who studied the optimal time for selling an asset, if the utility (reward) obtained by selling at the time $t$ and when the price is $z$ was given by

$$
g(t, z)=e^{-\beta t}(z-1)^{+}
$$

the time-price process being

$$
X_{t}=\left(t, Z_{t}\right)
$$

where $Z_{t}$ is a one-dimensional geometric Brownian motion, i.e.

$$
Z_{t}=z \exp \left(\alpha t+\beta B_{t}\right)
$$

for some constants $\alpha, \beta$ and a Brownian motion $B_{t}$. 
A rigorous proof of the high contact principle in this case was given by McKean [7], in the Appendix to the same article.

Subsequently the high contact principle for time-space Brownian/ geometric Brownian motion has been studied by several authors. See e.g. Bather [1], Merton [8], Van Moerbeke [13]. A proof for more general onedimensional diffusions has been given by Shiryaev [12].

For general diffusions in $\mathbb{R}^{n}$ less is known. The most general results seem to be due to A Friedman [6], and A. Bensoussan and J. L. Lions [2]. They prove regularity results for solutions of variational inequalities. The high contact principle then follows from the equivalence between optimal stopping problems and certain variational inequalities. For example, we mention the following result:

THEOREM B ([6], Theorem 8.1):

Suppose the generator $A$ of $X_{t}$, which is given by

$$
A f(x)=\sum_{i=1}^{n} b_{i}(x) \frac{\partial f}{\partial x_{i}}+\sum_{i, j=1}^{n} a_{i j}(x) \frac{\partial^{2} f}{\partial x_{i} \partial x_{j}}
$$

where $a=\left[a_{i, j}\right]=\frac{1}{2} \sigma \sigma^{T}\left(\sigma^{T}\right.$ being the transposed of $\sigma$ ) satisfies:

$A$ is uniformly elliptic, i.e. there exists $\beta>0$ such that $\xi^{t} a \xi \geq \beta|\xi|^{2} \quad$ for all $\xi \in \mathbb{R}^{n}$

$$
a_{i j} \text { and } \frac{\partial a_{i j}}{\partial x_{t}} \text { are bounded functions on } \mathbb{R}^{n}
$$

$$
b_{i}(x)=\sum_{j=1}^{n} \frac{\partial a_{i j}}{\partial x_{j}}(x)
$$

Let $W^{k, p, \mu}$ denote the set of functions $u$ on $\mathbb{R}^{n}$ whose first $k$ weak derivatives exists and belong to $L_{l o c}^{p}$ and whose norm

$$
\|u\|_{k, p, \mu}=\left\{\sum_{|\alpha| \leq k} \int\left|e^{-\mu|x|} D^{\alpha} u(x)\right|^{p} d x\right\}^{1 / p}
$$


is finite. Then if $f, g \geq 0$ satisfy

$$
g \in W^{2, p, \mu} \cap W^{2,2, \mu}
$$

$$
f \in W^{0, p, \mu} \cap W^{0,2, \mu} \text { for some } p>n
$$

then the solution $V(x)$ of the optimal stopping problem

$$
V(x):=\inf _{\tau} E^{x}\left[\int_{0}^{\tau} f\left(X_{t}\right) d t+g\left(X_{\tau}\right)\right]
$$

is continuous, $\nabla V$ is continuous and

$$
\frac{\partial V}{\partial x_{i}}=\frac{\partial g}{\partial x_{i}} \text { on } S
$$

where

$$
S:=\{x: V(x)=g(x)\},
$$

(It is also assumed that

$$
T:=\inf \left\{t>0 ; X_{t} \in S\right\}<\infty \text { a.s. } Q^{x}
$$

for all $x$ ).

Note that although the statement of the problem (1.12) appears to be more general than our original problem (1.2), it can be reduced to our case by considering the diffusion $Y_{t}$ given by

$$
d Y_{t}=\left[\begin{array}{l}
d X_{t} \\
d Z_{t}
\end{array}\right]=\left[\begin{array}{l}
b\left(X_{t}\right) \\
f\left(X_{t}\right)
\end{array}\right] d t+\left[\begin{array}{cc}
\sigma\left(X_{t}\right) & 0 \\
0 & 1
\end{array}\right] d \tilde{B}_{t}, Y_{0}=(z, x)
$$

where $\tilde{B}_{t}=\left(B_{1}(t), \ldots, B_{m}(t), B_{m+1}(t)\right)$ is $(m+1)$-dimensional Brownian motion, and the optimal stopping problem

$$
h^{*}(y)=h^{*}(x, z)=\sup _{\tau} E^{x, z}\left[h\left(Y_{\tau}\right)\right]
$$

with $h(x, z)=z+g(x)$. We will return to this in the end of $\S 3$.

It is natural to ask if there is a more direct approach to the high contact principle than via the formidable machinery of variational inequalities. The 
purpose of this paper is to point out that such a direct approach exists. Moreover, it gives the high contact conclusion with weaker assumptions than what appears to be known earlier.

In $\S 2$ we show how a simple argument can be used to give a weak but general version of the high contact principle. Then in $\S 3$ we apply results about stochastic waves due to Dynkin and Vanderbei [5] to obtain a strong version.

\section{$\S 2$. A weak but general version}

In this section we show that the high contact principle is basically a consequence of the strong Markov property, modulo some (non-tractable) differentiability conditions. First we introduce some notation: (As usual $C^{k}$ denotes the family of functions whose derivatives of order up to $k$ are continuous). Suppose $X_{t}, g, D$ are as in Theorem A. Assume that $\partial D$ locally (in a relatively open subset $W$ of $\partial D$ ) is the graph of a function

$$
x_{j}=\eta(y), \text { where } y=\hat{x}=\left(x_{1}, \ldots, x_{j-1}, x_{j}, \ldots, x_{n}\right) \in V \subset \mathbb{R}^{n-1}
$$

where $V$ is open, and that $D$ near $W$ is situated "below" $W$ viewed along the $x_{j}$-axis. We introduce the following perturbations of $D$ at $W$ : Let $V_{0}$ be an open set with $\bar{V}_{0} \subset V$, and for each $\theta \in(-1,1)$ let $\alpha_{\theta}: V \rightarrow \mathbb{R}$ satisfy

(i) $\alpha_{\theta}=0$ outside $V_{0}$

(ii) $\alpha_{0}=0$

(iii) $(\theta, y) \rightarrow \alpha_{\theta}(y)$ is $C^{1}$ on $(-1,1) \times V$ and

$$
\alpha_{\theta}^{\prime}(y)=\frac{d}{d \theta} \alpha_{\theta}(y)>0 \text { on }(-1,1) \times V_{0} .
$$

Let $D_{\theta}$ denote the domain obtained by replacing $\partial D$ in $W$ by the graph of

$$
x_{j}=\eta(y)+\alpha_{\theta}(y) ; y \in V
$$

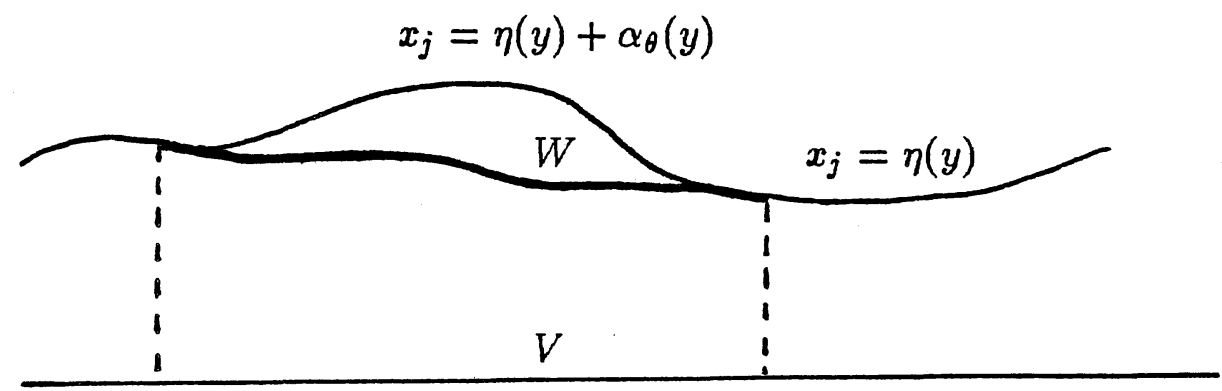


Let $W_{\theta}$ denote the corresponding part of $\partial D_{\theta}$ and let $\tau_{\theta}=\tau_{D_{\theta}}$ be the first exit time from $D_{\theta}$ (writing $\tau_{0}=\tau$ ).

Finally, if $h$ is a function on $\mathbb{R}^{n}$ we let $\overline{D_{j}} h(x)$ denote the left-sided partial derivative of $h$ with respect to $x_{j}$, i.e.

$$
\overline{D_{j}} h(x)=\lim _{\varepsilon \rightarrow 0^{+}} \frac{g(x)-g\left(x-\varepsilon e_{j}\right)}{-\varepsilon}
$$

(if the limit exists), where $e_{j}$ is the $j$ th unit vector in $\mathbb{R}^{n} ; 1 \leq j \leq n$.

THEOREM 1 (Weak version I of the high contact principle).

Let $X_{t}, g, D$ be as in Theorem A. Suppose that, locally at $W \subset \partial D$, $\partial D$ has the form (2.1) and $D$ is situated below this graph there. Moreover, assume the following:

The functions $x \rightarrow D_{j} g(x), x \rightarrow D_{j} g^{*}(x)$ are bounded and uniformly continuous in $G \cap D$, for some $\mathbb{R}^{n}$-neighborhood $G$ of $W$

All the points $x \in W$ are $X$-regular boundary points of $D$

i.e. $P^{x}[\tau=0]=1$ for all $x \in W$.

The function $F(\theta)=E^{x}\left[g\left(X_{\tau_{\theta}}\right)\right]$ is differentiable at

$$
\theta=0, \text { for all } x \in D
$$

Then

$$
\begin{aligned}
& E^{x}\left[\overline{D_{j}} g\left(X_{\tau}\right) \cdot \alpha_{0}^{\prime}\left(\hat{X}_{\tau}\right) \cdot K_{0}\right] \\
& \quad=E^{x}\left[\overline{D_{j}} g^{*}\left(X_{\tau}\right) \cdot \alpha_{0}^{\prime}\left(\hat{X}_{\tau}\right) \cdot K_{0}\right], x \in D
\end{aligned}
$$

where

$$
K_{\theta}=\chi_{x_{\tau_{\theta}} \in W_{\theta}} \text { for } \theta \in(-1,1),
$$

$\chi$ being the indicator function (characteristic function).

\section{Proof.}

If $\theta<0$ then $\tau_{\theta}<\tau$ so by the strong Markov property we have

$$
\begin{aligned}
& E^{x}\left[g\left(X_{\tau}\right) \cdot K_{\theta}\right]=E^{x}\left[E^{x}\left[g\left(X_{\tau}\right) \cdot K_{\theta} \mid \mathcal{F}_{\tau_{\theta}}\right]\right. \\
= & E^{x}\left[K_{\theta} \cdot E^{X_{\tau_{\theta}}}\left[g\left(X_{\tau}\right)\right]\right]=E^{x}\left[K_{\theta} \cdot g^{*}\left(X_{\tau_{\theta}}\right)\right]
\end{aligned}
$$


This gives, with $\theta<0$ and $\Delta \alpha=\alpha_{0}\left(\hat{X}_{\tau_{\theta}}\right)-\alpha_{\theta}\left(\hat{X}_{\tau_{\theta}}\right)$,

$$
\begin{aligned}
& F(0)-F(\theta)=E^{x}\left[g\left(X_{\tau}\right) \cdot K_{\theta}\right]-E^{x}\left[g\left(X_{\tau_{\theta}}\right) \cdot K_{\theta}\right] \\
& =E^{x}\left[\left(g\left(X_{\tau}\right)-g\left(X_{\tau_{\theta}}+\Delta \alpha \cdot e_{j}\right)\right) K_{\theta}\right]+E^{x}\left[\left(g\left(X_{\tau_{\theta}}+\Delta \alpha \cdot e_{j}\right)-g\left(X_{\tau_{\theta}}\right)\right) \cdot K_{\theta}\right] \\
& =-E^{x}\left[\left(g^{*}\left(X_{\tau_{\theta}}+\Delta \alpha \cdot e_{j}\right)-g^{*}\left(X_{\tau_{\theta}}\right)\right) K_{\theta}\right]+E^{x}\left[\left(g\left(X_{\tau_{\theta}}+\Delta \alpha \cdot e_{j}\right)-g\left(X_{\tau_{\theta}}\right)\right) \cdot K_{\theta}\right] \\
& =-E^{x}\left[D_{j} g^{*}\left(X_{\tau_{\theta}}+\mu \cdot e_{j}\right) \cdot \Delta \alpha \cdot K_{\theta}\right]+E^{x}\left[D_{j} g\left(X_{\tau_{\theta}}+\mu^{\prime} \cdot e_{j}\right) \cdot \Delta \alpha \cdot K_{\theta}\right],
\end{aligned}
$$

where $0 \leq \mu, \mu^{\prime} \leq \Delta \alpha$. Since $F(\theta)$ is maximal for $\theta=0$ and $F$ is differentiable at $\theta=0$, we get from this that

$0=\lim _{\theta \rightarrow 0^{-}} \frac{F(0)-F(\theta)}{-\theta}=-E^{x}\left[D_{j}^{-} g^{*}\left(X_{\tau}\right) \cdot \alpha_{0}^{\prime}\left(\hat{X}_{\tau}\right) K_{0}\right]+E^{x}\left[D_{j}^{-} g\left(X_{\tau}\right) \cdot \alpha_{0}^{\prime}\left(\hat{X}_{\tau}\right) \cdot K_{0}\right]$,

as claimed.

In the previous result the condition (2.4) can be replaced by a one-sided differentiability condition on the $X$-harmonic extensions:

Define $\tilde{g}^{\theta}(x)$ to be the $X$-harmonic extension of $g \mid \partial D_{\theta}$ to $D_{\theta}$, i.e.

$$
\tilde{g}^{\theta}(x)=E^{x}\left[g\left(X_{\tau_{\theta}}\right)\right] ; \theta \in(-1,1)
$$

THEOREM 2 (Weak version II of the high contact principle)

Let $X_{t}, g, D$ be as in Theorem A. Suppose that, locally at $W \subset \partial D, \partial D$ has the form (2.1) and D is situated below this graph there. Assume that there exists an $\mathbb{R}^{n}$-neighborhood $G$ of $W$ such that

$$
x \rightarrow D_{j} g(x) \text { is bounded and continuous in } G
$$

and

(2.8) $(\theta, x) \rightarrow D_{j} \tilde{g}^{\theta}(x)$ is uniformly continuous for $x \in G \cap D_{\theta}, \theta \in(-1,1)$.

Moreover, assume that all the points of $W_{\theta}$ are $X$-regular boundary points of $D_{\theta}$, i.e.

$$
P^{x}\left[\tau_{\theta}=0\right]=1 \text { for all } x \in W_{\theta}, \theta \in(-1,1) .
$$

Then

$$
E^{x}\left[D_{j} g\left(X_{\tau}\right) \cdot \alpha_{0}^{\prime}\left(X_{\tau}\right) \cdot K_{0}\right]=E^{x}\left[D_{j}^{-} g^{*}\left(X_{\tau}\right) \cdot \alpha_{0}^{\prime}\left(X_{\tau}\right) \cdot K_{0}\right], x \in D
$$


Proof. We proceed as in the proof of Theorem 1:

If $\theta_{1}<\theta_{2}$ then $\sigma:=\tau_{\theta_{1}} \leq \zeta:=\tau_{\theta_{2}}$ so by the strong Markov property we get as in (2.6)

$$
E^{x}\left[g\left(X_{\zeta}\right) \cdot K_{\theta_{1}}\right]=E^{x}\left[\tilde{g}^{\theta_{2}}\left(X_{\sigma}\right) \cdot K_{\theta_{1}}\right]
$$

Therefore, if $\Delta \alpha=\alpha_{\theta_{2}}\left(\hat{X}_{\sigma}\right)-\alpha_{\theta_{1}}\left(\hat{X}_{\sigma}\right)$ the same argument as above gives

$$
F\left(\theta_{2}\right)-F\left(\theta_{1}\right)=E^{x}\left[g\left(X_{\zeta}\right)\right]-E^{x}\left[g\left(X_{\sigma}\right)\right]
$$

$$
=-E^{x}\left[D_{j} \tilde{g}^{\theta_{2}}\left(X_{\sigma}+\xi e_{j}\right) \cdot \Delta \alpha \cdot K_{\theta_{1}}\right]+E^{x}\left[D_{j} g\left(X_{\theta}+\xi^{\prime} \cdot e_{j}\right) \cdot \Delta \alpha \cdot K_{\theta_{1}}\right],
$$

where $0 \leq \xi, \xi^{\prime} \leq \Delta \alpha$. In particular, $F$ is a continuous function of $\theta$.

Therefore, since $F(\theta)$ is maximal for $\theta=0$ we can find sequences

$$
\theta_{1}(k) \leq 0 \leq \theta_{2}(k)
$$

such that

$$
\begin{gathered}
\theta_{2}(k)-\theta_{1}(k) \neq 0, \theta_{2}(k)-\theta_{1}(k) \rightarrow 0 \text { and } \\
F\left(\theta_{2}(k)\right)-F\left(\theta_{1}(k)\right)=0 \text { for all } k .
\end{gathered}
$$

By (2.12) this gives, by dividing by $\theta_{2}(k)-\theta_{1}(k)$ and letting $k \rightarrow \infty$,

$$
0=-E^{x}\left[\overline{D_{j}^{-}} g^{*}\left(X_{\tau}\right) \cdot \alpha_{0}^{\prime}\left(X_{\tau}\right) \cdot K_{0}\right]+E^{x}\left[D_{j} g\left(X_{\tau}\right) \cdot \alpha_{0}^{\prime}\left(X_{\tau}\right) \cdot K_{0}\right]
$$

which is (2.10).

Letting $x \rightarrow \partial D$ we deduce the usual conclusion of the high contact principle:

COROLLARY 1. Suppose the conditions of Theorem 1 or Theorem 2 are satisfied and that a point $\bar{x} \in W$ satisfies:

There exists a sequence $\left\{x_{k}\right\} \subset D$ such that $x_{k} \rightarrow \bar{x}$ and

$$
Q^{x_{k}}\left[\left|X_{\tau}-\bar{x}\right|<\varepsilon\right] \rightarrow 1 \text { as } k \rightarrow \infty
$$

for all $\varepsilon>0$. Then

$$
D_{j} g(\bar{x})=\overline{D_{j}} g^{*}(\bar{x})
$$

REMARK Condition (2.13) holds for all $\bar{x} \in W$ if (for example) $X_{t}$ is a strong Feller process. (See Dynkin [4, Th. 13.3]). And for this to be the case it is sufficient that the generator $A$ of $X_{t}$ is uniformly elliptic, i.e. that (1.6) holds. 


\section{§3. Application of stochastic waves}

One of the major drawbacks of the approach in $\S 2$ was the intractable condition (2.4) (or (2.8)). We will now find conditions on the generator $A$ which will ensure that (2.4) holds. This is achieved by applying a result of Dynkin and Vanderbei [5] about stochastic waves. We first summarize this theory. For details see [5].

Let $\varphi$ be a real, measurable function on $\mathbb{R}^{n}$. For $t \geq 0$ define

$$
T_{t}=\inf \left\{s>0 ; \quad \varphi\left(X_{s}\right)>\varphi\left(X_{0}\right)+t\right\}
$$

Assume that

$$
\text { (i) } T_{t}<\infty \text { for all } t
$$

(ii) $\varphi\left(X_{t}\right)$ is continuous in $t$

(iii) $T_{0}=0$ a.s. $Q^{x}$ for all $x$.

Define $\tilde{X}_{t}=X_{T_{t}}, \tilde{\mathcal{M}}_{t}=\mathcal{M}_{T_{t}}$ and $\tilde{\theta}_{t}=\theta_{T_{t}}$. Then $\left(\tilde{X}_{t}, \tilde{\mathcal{M}}_{t}, Q^{x}, \tilde{\theta}_{t}\right)$ is a strong Markov process, called the stochastic wave corresponding to $X$ and $\varphi$. The generator $\tilde{A}$ of $\tilde{X}_{t}$ is defined by

$$
\tilde{A} f=\lim _{t \rightarrow 0} \frac{E \cdot\left[f\left(\tilde{X}_{t}\right)\right]-f}{t}
$$

the limit being in the uniform topology on $\mathbb{R}^{n}$. We let $\tilde{\mathcal{D}}$ denote the set of functions $f$ for which the limit (3.2) exists. We say that $f \in \tilde{\mathcal{D}}_{x}$ if there exists $f^{*} \in \tilde{\mathcal{D}}$ such that $f^{*}=f$ in a neighborhood of $x$ in the topology generated by $\varphi$. In that case we put $\tilde{A} f(x)=\tilde{A} f^{*}(x)$.

If $V \subset \mathbb{R}^{n}$ is open let $C^{2, \lambda}(V)$ denote the set of functions with partial derivatives up to order 2 which are Hölder continuous with some exponent $\lambda>0$. The main result of [5] then gives

THEOREM C [5]. Let $\varphi$ satisfy (3.1) and assume that

$$
\left.\varphi \in C^{2, \lambda}\left(\mathbb{R}^{n}\right)\right) \text { and } \nabla \varphi \neq 0 \text { in } \mathbb{R}^{n}
$$

For some $x \in \mathbb{R}^{n} \quad$ the set $G^{x}=\{y ; \varphi(y) \leq \varphi(x)\} \quad$ is bounded

The generator $A$ of $X_{t}$ is uniformly elliptic (i.e. (1.6) holds). 
Then every $f \in C^{2, \lambda}\left(\mathbb{R}^{n}\right)$ belongs to $\tilde{\mathcal{D}}_{x}$ and

$$
\tilde{A} f(x)=|\nabla \varphi(x)|^{-1}\left(\frac{\partial f}{\partial n}(x)+H f(x)\right)
$$

where

$H f(x)$ is the interior normal derivative at $x$ of the harmonic extension to $G^{x}$ of $f \mid \partial G^{x}$ and $\frac{\partial f}{\partial n}$ denotes the derivative of $f$ in the direction of the outer normal.

\section{Localization}

Before we apply this to the optimal stopping problem let us observe that we may localize the problem of high contact as follows:

Let $G$ be a bounded open set and define $\hat{D}=G \cap D$. Modify $g$ to a function $g_{1}$ satisfying the following conditions:

(a) $g_{1}=g^{*}$ outside $\hat{D}$

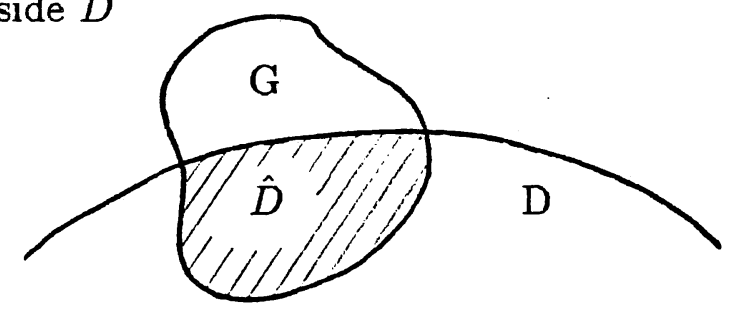

(b) $g_{1}=g$ in a neighborhood of $\partial D \cap G$

(c) $g \leq g_{1}<g^{*}$ on $\hat{D}$

(d) $g_{1}$ is continuous.

Then note that $g^{*}$ is an $X$-superharmonic majorant of $g_{1}$ so if $\hat{g}_{1}$ denotes the least superharmonic majorant of $g_{1}([10]$, Ch. X) we have

$$
g_{1}^{*} \geq g^{*} \geq \hat{g}_{1}=g_{1}^{*}
$$

and we conclude that $g^{*}=g_{1}^{*}$. Consequently $\hat{D}$ is the continuation region for $g_{1}$. So if we want to investigate $\overline{D_{j}} g$ and $\overline{D_{j}^{-}} g^{*}$ near a point in $\partial D$ we may reduce/modify $D$ outside a neighborhood of this point as desired.

In particular, assume that, locally at $W \subset \partial D, \partial D$ has the form (2.1), i.e.

$$
x_{j}=\eta(\hat{x}) \quad ; \hat{x}=\left(X_{1}, \ldots, x_{j-1}, x_{j+1}, \ldots, x_{n}\right) \in V \subset \mathbb{R}^{n-1}
$$


(with $D$ situated "below" $W$ ) where we now assume that

$$
\eta \in C^{2, \lambda}(V) \text { for some } \lambda>0
$$

Then by modifying $D$ outside an $\mathbb{R}^{n}$-neighorhood of $W$ we may assume that $D$ has the form

$$
D=\{x ; \varphi(x)<0\}
$$

where $\varphi$ is a function in $C^{2, \lambda}\left(\mathbb{R}^{n}\right)$ such that

$$
\varphi(x)=x_{j}=\eta(\hat{x}) \quad \text { for } x \in D \quad \text { near } W
$$

and

$$
\{y ; \varphi(y) \leq M\} \quad \text { is bounded for all } M, x
$$

This enables us to combine Theorem $\mathrm{A}$ with Theorem $\mathrm{C}$ and we obtain the following:

THEOREM 3 (Strong version I of the high contact principle)

Let $X_{t}, g, D$ be as in Theorem A. Assume that there exists $\lambda>0$ such that, locally at $W \subset \partial D, \partial D$ has the form

$$
x_{j}=\eta(\hat{x}) ; \hat{x}=\left(x_{1}, \ldots, x_{j-1}, x_{j+1}, \ldots, x_{n}\right) \in V \subset \mathbb{R}^{n-1}
$$

where $\eta \in C^{2, \lambda}(V)$ and let $\varphi \in C^{2, \lambda} \mathbb{R}^{n}$ ) be the corresponding level function as in (3.8). Moreover, assume that

(3.10) The generator $A$ of $X_{t}$ is uniformly elliptic (i.e. (1.6) holds)

and

$$
g \in C^{2, \lambda}\left(\mathbb{R}^{n}\right)
$$

Then

$$
D_{j} g(x)=\overline{D_{j}} g^{*}(x) \text { for } x \in W
$$

Proof. By localization we may assume that $D$ has the form

$$
D=\{x ; \varphi(x)<0\}
$$


(By uniform ellipticity $g^{*} \in C^{2, \lambda}(D)$ so the modified $g_{1}$ can be chosen in $C^{2, \lambda}\left(\mathbb{R}^{n}\right)$ too). By uniform ellipticity we obtain that (3.1) holds so we can apply Theorem C: We now consider the following analogue $H(t)$ of the function $F(\theta)$ of Theorem 1:

Define, for a fixed $x \in D$,

$$
H(t)=E^{x}\left[g\left(X_{T_{t}}\right)\right]=E^{x}\left[g\left(\tilde{X}_{t}\right)\right] ; t \geq 0
$$

By Dynkin's formula ([3, p. 133]) we have

$$
H(t)=g(x)+E^{x}\left[\int_{0}^{t} \tilde{A} g\left(\tilde{X}_{s}\right) d s\right]
$$

In particular, $H$ is a differentiable function of $t$. Moreover, $H(t)$ is maximal for the value $t_{0}$ of $t$ which gives $\varphi(x)+t_{0}=0$. Therefore $H^{\prime}\left(t_{0}\right)=0$.

Proceeding as in the proof of Theorem 1 we get for $t<t_{0}$

$$
H\left(t_{0}\right)-H(t)=-E^{x}\left[\nabla g^{*}\left(X_{T_{t}}+\delta \gamma_{t}\right) \cdot \gamma_{t}\right]+E^{x}\left[\nabla g\left(X_{T_{t}}+\delta^{\prime} \gamma_{t}\right) \cdot \gamma_{t}\right]
$$

(for some $\delta, \delta^{\prime} \in[0,1]$ ) where $\gamma_{t}=\left(t_{0}-t\right) \cdot e_{j}$ near $W$, and elsewhere $\gamma_{t}$ is the vector parallel to $\nabla \varphi\left(X_{T_{t}}\right)$ such that

$$
X_{T_{t}}+\gamma_{t} \in \partial D\left(\text { i.e. } \gamma_{t} \approx\left(t_{0}-t\right) \cdot \frac{\nabla \varphi}{|\nabla \varphi|^{2}}\right)
$$

Dividing by $t_{0}-t$ and letting $t \uparrow t_{0}$ we obtain

$$
\begin{aligned}
0 & =E^{x}\left[\left(\frac{\partial g^{*}}{\partial n_{i}}\left(X_{\tau}\right)+\frac{\partial g}{\partial n_{0}}\left(X_{\tau}\right)\right) \cdot\left(1-K_{0}\right)\right] \\
& +E^{x}\left[\left(-\overline{D_{j}^{-}} g^{*}\left(X_{\tau}\right)+D_{j} g\left(X_{\tau}\right)\right) \cdot K_{0}\right]
\end{aligned}
$$

where $\frac{\partial}{\partial n_{i}}$ denotes the derivative in the direction of the inner normal to $D$. (It follows from Theorem $36 . \mathrm{V}$ in [9] that $\frac{\partial g^{*}}{\partial n_{i}}$ and $\bar{D}_{j}^{-} g^{*}$ exist and are bounded at $\partial D)$.

Letting $x$ in (3.12) approach a given point in $W$ we obtain the conclusion of Theorem 3 .

Finally we show how the more general optimal stopping problem

$$
\gamma^{*}(x)=\sup _{\tau} E^{x}\left[\int_{0}^{\tau} f\left(X_{s}\right) d s+g\left(X_{\tau}\right)\right]
$$


can be reduced to the case considered above. Let $Y_{t}$ be as in (1.14), so that

$$
Y_{t}=\left[\begin{array}{c}
X_{t} \\
Z_{t}
\end{array}\right] \text { with } Z_{t}=\int_{0}^{t} f\left(X_{s}\right) d s+B_{m+1}(t)
$$

Define

$$
h(y)=h(x, z)=z+g(x) .
$$

Then (since $E\left[B_{m+1}^{0}(\tau)\right]=0$ for all $\tau$ )

$$
\begin{aligned}
h^{*}(y) & =\sup _{\tau} E^{y}\left[h\left(Y_{\tau}\right)\right]=\sup _{\tau} E^{x, z}\left[Z_{\tau}+g\left(X_{\tau}\right)\right] \\
& =\sup _{\tau} E^{x, z}\left[z+\int_{0}^{\tau} f\left(X_{s}\right) d s+B_{m+1}^{0}(\tau)+g\left(X_{\tau}\right)\right] \\
& =z+\sup _{\tau} E^{x}\left[\int_{0}^{\tau} f\left(X_{s}\right) d s+g\left(X_{\tau}\right)\right]=z+\gamma^{*}(x)
\end{aligned}
$$

If the generator $A$ of $X_{t}$ is uniformly elliptic, then so is the generator of $Y_{t}$. Therefore, if $f$ is "reasonable" we can apply Theorem 3 to. $Y_{t}$. We get the following conclusion:

THEOREM 4 (Strong version II of the high contact principle)

Let $X_{t}$ and $g$ be as in Theorem 3. Let $f$ be a Lipschitz continuous function with at most linear growth. Define

$$
\begin{aligned}
\gamma^{*}(x) & =\sup _{\tau} E^{x}\left[\int_{0}^{\tau} f\left(X_{s}\right) d s+g\left(X_{\tau}\right)\right], \\
D & =\left\{x ; g(x)<\gamma^{*}(x)\right\} .
\end{aligned}
$$

Then at any point $x$ of $\partial D$ where $\partial D$ locally has the form (3.9) of Theorem 3 we have

$$
D_{j} g(x)={\overline{D_{j}}}^{*} g^{*}(x)
$$

Acknowledgement.

This paper was written while the author was visiting the University of California at San Diego (UCSD). I wish to thank UCSD and the Mathematics Department there for their hospitality. 


\section{REFERENCES}

[1] J. A. Bather: Optimal stopping problems for brownian motion. Advances in Appl. Prob. 2 (1970), 259-286.

[2] A. Bensoussan \& J. L. Lions: Applications of Variational Inequalities in Stochastic Control. North-Holland 1982.

[3] E. B. Dynkin: Markov Processes, Vol. I. Springer-Verlag 1965.

[4] E. B. Dynkin: Markov Processes, Vol. II. Springer-Verlag 1965.

[5] E. B. Dynkin \& R. J. Vanderbei: Stochastic waves. Transactions Amer. Math. Soc. 275 (1983), 771-779.

[6] A. Friedman: Stochastic Differential Equations and Applications, Vol. II. Academic Press 1976.

[7] H. P. McKean: A free boundary problem for the heat equation arising from a problem of mathematical economics. Industrial managem. review 6 (1965), 32-39.

[8] R. C. Merton: The theory of rational option pricing. Bell J. of Economic \& Management Science 4 (Spring) (1973), 141-183.

[9] C. Miranda: Partial Differential Equations of Elliptic Type. (2. ed.) Springer-Verlag 1970.

[10] B. Øksendal: Stochastic Differential Equations (2. ed.) Springer-Verlag 1989.

[11] P. A. Samuelson: Rational theory of warrant pricing. Industrial managem. review 6 (1965), 13-32.

[12] A. N. Shiryaev: Optimal Stopping Rules. Springer-Verlag 1978.

[13] P. Van Moerbeke: An optimal stopping problem with linear reward. Acta Mathematica 132 (1974), 111-151.

\section{Current address:}

Dept. of Mathematics

University of California, San Diego

La Jolla, CA 92093

USA

Dept. of Mathematics

University of Oslo

Box 1053 Blindern, N-0316 Oslo 3

NORWAY 